\title{
Location of low-cost blood collection and distribution centres in Thailand
}

\author{
Pornpimol Chaiwuttisak ${ }^{\mathrm{a}, *}$, Honora Smith ${ }^{\mathrm{a}}$, Yue $\mathrm{Wu}^{\mathrm{a}}$, Chris Potts ${ }^{\mathrm{a}}$, Tasanee \\ Sakuldamrongpanich ${ }^{\mathrm{b}}$, Somchai Pathomsiric ${ }^{\mathrm{c}}$ \\ ${ }^{a}$ CORMSIS, University of Southampton, Highfield, Southampton, SO17 1BJ, UK \\ ${ }^{b}$ National Blood Centre, the Thai Red Cross Society, Bangkok, Thailand \\ ${ }^{c}$ Civil and Environmental Engineering department, Mahidol University, Thailand
}

\begin{abstract}
Decision making on facility locations for blood services has an impact on the efficiency of supply chain and logistics systems. In the blood supply chain operated by the Thai Red Cross Society (TRCS), problems are faced with amounts of blood collected in different provinces of Thailand being insufficient to meet demand. At the present time, TRCS operates one National Blood Centre in the capital and twelve Regional Blood Centres in different provinces to collect, prepare, test, and distribute safe blood. A proposal has been made to extend this network of blood centres using low-cost collection and distribution centres. Increasing numbers of fixed collection sites can improve access for donors. In addition, some facilities will be able to perform preparation and storage for blood that hospitals can receive directly. This paper addresses the selection of sites for two types of facility, either a blood donation room only or donation room with a distribution centre. A range of investment budgets is investigated to inform the strategic plan of this non-profit organisation. We present a novel binary integer programming model for this location-allocation problem based on objectives of improving the supply of blood products while reducing costs of transportation. Computational results are reported, using real life data, that are of practical importance to decision makers.
\end{abstract}

(C) 2014 Published by Elsevier Ltd.

Keywords: Blood supply chain, Location analysis, Developing countries

\section{Introduction}

The locations of suppliers, processing plants and distribution centres are critical to the efficient running of any supply chain network: this is especially important when transporting perishable products such as blood. Moreover, the essential nature of the blood supply chain in delivering blood products to hospitals brings special problems in developing countries such as Thailand where budgets are limited for setting up service facilities. This paper introduces a new location-allocation model for the siting of low-cost blood collection and distribution centres that are planned to provide an extended service to an existing network of regional blood centres in Thailand.

\footnotetext{
${ }^{*}$ Corresponding author.

Email address: molchaiwuttisak@outlook.com (Pornpimol Chaiwuttisak)
} 
Thailand's one national and twelve regional blood centres provide the full range of screening and testing services needed to ensure that safe blood products can be delivered to the nation's hospitals. Located in large cities, these centres perform the triple functions of blood collection, testing and distribution. However, donor supply remains insufficient for the growing needs of Thailand's hospitals, and budgets are constrained for the construction of new regional blood centres that could attract more blood donors. New low-cost blood service facilities are therefore planned to extend the supply side and also the distribution side of the blood supply chain, without incurring the costs of providing full testing and screening functions.

Two types of low-cost blood service facilities are planned, subject to financial constraints. The first type consists only of a donation room where blood can be collected from walk-in donors: such fixed facilities are best located in the large cities where donation is most likely to occur. Blood collected from such donation rooms must be transported to regional blood facilities for testing and distribution to hospitals. The second type of facility combines a donation room with a distribution centre where blood can be prepared for transportation directly to hospitals. From this second type of facility, samples of blood donated at these low-cost centres must be transported to the regional blood centres for screening checks before blood products can be distributed.

This paper presents locational analysis for these two types of facility, and the allocation of demand to either such new facilities or existing blood centres. Location-allocation modelling has a research base for a variety of applications in the healthcare arena. For example, the classical Maximal Covering Location Problem, formulated originally by Church and ReVelle [1], has been widely employed in emergency service applications to maximise coverage of demand points within a predetermined distance or travel time. Similarly, variations on the $p$-Median Model $[2,3]$ have been proposed to locate healthcare facilities according to minimised demand-weighted average travel distance, given a fixed number of $p$ facilities [4].

The novel location-allocation model proposed here finds optimal numbers and locations within budgetary constraints for both types of service facilities according to a weighted combination of three criteria. The first criterion maximises the estimated supply of blood, according to likely donations at candidate locations. Secondly, total distances travelled are minimised in transporting blood or blood samples from either type of facility to an allocated regional blood centre. Thirdly, total distances travelled are minimised in transporting blood from distribution centres or regional blood centres to hospitals, weighted by amounts of blood carried. Constraints are introduced on travel times from donation rooms to regional blood centres, to avoid blood quality deterioration in unrefrigerated conditions.

The rest of the paper is organised as follows. Section 2 describes the blood collection and distribution system in Thailand. Section 3 reviews relevant literature on location allocation problems in the blood supply chain. In Section 4, the problem is defined and the integer linear programming (ILP) model is proposed. The results of analysis based on real-world data are presented and discussed in Section 5. Finally, conclusions and directions for future research are given in Section 6.

\section{Problem background: the blood collection and distribution system in Thailand}

The Thai Red Cross Society (TRCS) has responsibility for blood collection and delivery services throughout the seventy six provinces of Thailand. Key to these services are the National Blood Centre (NBC) in Bangkok and twelve Regional Blood Centres (RBCs) located in different provinces of the country, all of which offer facilities for donation of blood, screening and distribution. Both the NBC and RBCs distribute blood to hospitals in all provinces, as determined by government administration. The ultimate mission of the TRCS is ensuring sufficient safe blood supply and the same quality standards across the country.

The main activities of the NBC and RBCs are illustrated in Figure 1. These activities are typical of blood centres in other countries. Blood screening and tests carried out include tests for blood groups and for infections such as Hepatitis, Syphilis and Human Immunodeficiency Virus (HIV). Whole blood is centrifuged into blood products in the form of red blood cells, plasma, and platelets. Safe blood is stored for subsequent distribution to hospitals. In addition, the blood centres perform an educational role in imparting knowledge and understanding in their localities of the importance and benefits of blood donation. 


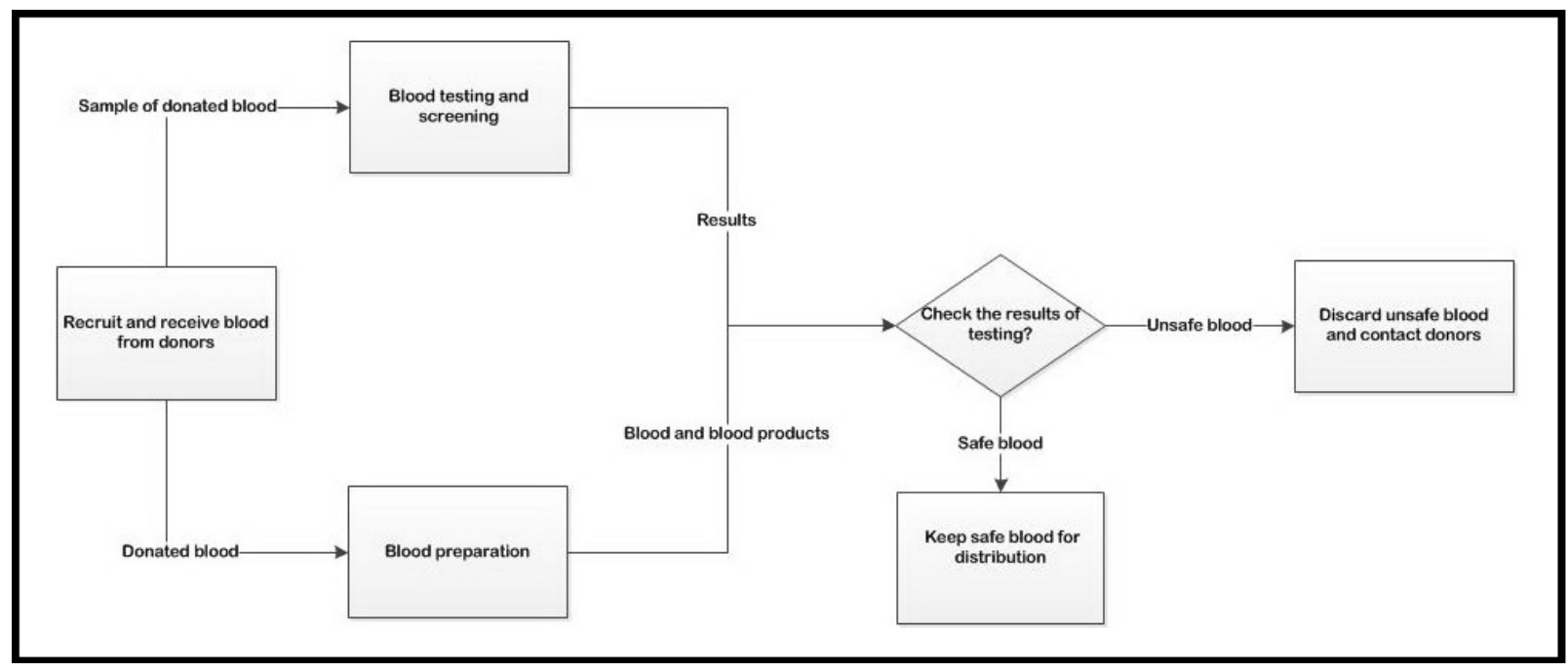

Figure 1: Main functions of a blood centre

The current blood supply chain and logistics process in Thailand can be seen in Figure 2. Walk-in donors can donate blood at donation sites run by the NBC and RBCs, including at mobile facilities known as bloodmobiles. Hospitals then collect blood products from the NBC and the RBCs using their own vehicles. In addition, there is exchange of blood and its products amongst hospitals. Hospitals may also collect additional blood from local donors, but samples must be sent to an RBC for safety and quality screening. Results are then reported to the hospitals before blood is transfused to patients. However, hospitals may incur high costs in sending blood samples for testing if the nearest RBC is at a distance.

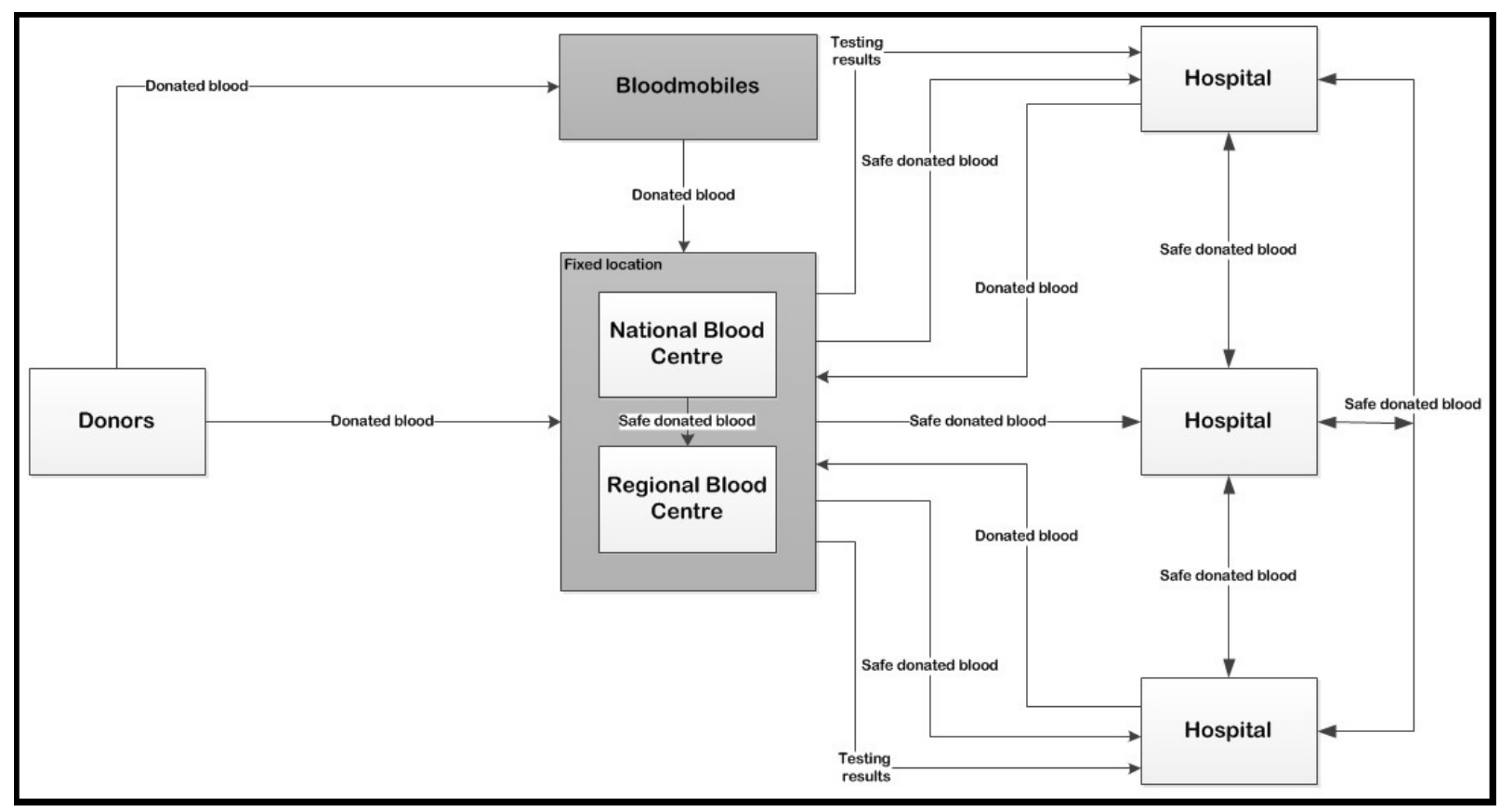

Figure 2: The current blood supply chain in Thailand 
Shortage of supply of quality blood products is the major problem confronted in Thailand, and in other developing countries. In Thailand, it is in the provinces remote from Bangkok that demand tends to exceed supply. The reverse occurs in the capital, necessitating the transportation of more than $40 \%$ of blood collected by the NBC in Bangkok out to areas of shortfall throughout the country. Blood is sent by public transportation: aeroplane, bus, and van depending on the available transportation modes and geographical locations of the provinces where the RBCs are located.

The TRCS has proposals which this paper seeks to support, for addressing weaknesses in the supply of quality blood products in the current blood supply chain. Ideally, more fully functioning RBCs would be established to increase the supply of quality blood products, but costs are prohibitive for these main screening and testing centres. The foundation of simpler fixed donation rooms is proposed to facilitate easy access for donors who may be unaware of the timing and place of bloodmobile sessions. In addition, hospitals in some provinces suffer significant costs in collecting blood from remote RBCs or the NBC, since they send their own vehicles to collect blood. Lengthy transportation may also have an effect on quality of blood products received. So, donation rooms with distribution centres are a second type of facility proposed to improve the logistics system. Such centres would have the ability to carry out all blood processing except the testing and screening stage. All donation rooms with or without distribution facilities would be allocated to blood centres for testing of blood in a timely manner.

Optimal numbers and locations for these two types of low-cost blood service facilities need to be determined under the budgetary constraints of the TRCS. For the first type of facility consisting just of a donation room, costs must be considered firstly of transporting the blood collected to regional blood facilities for testing and secondly of distribution to hospitals. From the second type of facility including distribution capabilities, costs are incurred transporting samples of blood to the regional blood centres for safety checks as well as costs of distribution to hospitals.

Budgetary constraints on development of both types of low-cost facility have to take into account land costs, building costs and equipment costs. Land costs vary throughout the provinces of Thailand. Building costs include labour costs, again varying throughout Thailand. The equipment costs for a type 2 facility, with equipment for preparing blood for distribution, are more expensive than for type 1 donation rooms alone.

\section{Literature Review}

In this section, we give an overview of operational research (OR) studies relevant to the location of blood service facilities and in particular to blood-related location-allocation problems.

The range of problems related to regional or community blood centres are categorised by Pierskalla [5]: which functions should be performed at blood centres, locations for these centres, the number of community blood centres that should be located in a region, and allocation of transfusion services to the community blood centres. Other problems of the blood supply chain that are highlighted by Pierskalla include the collection of blood, producing multiple products, inventory control, allocating blood to the hospitals, blood delivery to multiple sites, issuing policies and crossmatching policies. Also taking a wide view of the blood supply chain, Rautonen [6], in a study assessing the Finnish blood supply system, concludes that cooperation between blood services and both donors and hospitals can improve the national blood supply chain.

The Blood Transportation-Allocation Problem (BTAP) is defined by Or and Pierskalla [7] in terms of how many central blood banks to set up and in which locations, which hospitals should be allocated to each blood bank, and how to route the periodic supply distribution so that the total of system and transportation costs, both emergency and periodic, are at a minimum. This problem is a combination of the General Transportation Problem (GTP) and Location-Allocation Problem (LAP) with added considerations of emergency and periodic delivery costs. A heuristic algorithm is successfully used to provide solutions with data for the Chicago area.

Some complex problems of blood supply chain management have been decomposed into simpler sub-problems. Different objectives for each sub-problem are presented, with most papers focusing on 
minimising system costs, especially transportation costs. For example, Jacobs et al. [8] consider a facility relocation problem of blood services in the mid-Atlantic region of the American Red Cross (ARC) in Norfolk, Virginia. Two Integer Programming models for collection and distribution are used to evaluate the total transportation costs. The costs of collection include a labour cost, which is, however, excluded in the distribution model. Dessouky et al. [9] present separate models for location of facilities and vehicle routing in emergency distribution of supplies; this study is motivated by supply of medicine, but is relevant to blood supplies.

Similarly, a sequence of models are designed by Şahin et al. [10] to address the hierarchical locationallocation problem of the Turkish Red Crescent Society in organising blood supplies. The problem is formulated as three sub-problems. Firstly, a $p q$-median location model is proposed to minimize the total of population-weighted distances travelled. Next, a set-covering model is used to find locations for blood donation facilities. The final model based on integer programming is formulated to determine distribution of mobile units among the service regions.

Contrastingly, a multi-objective goal programming approach is employed by Çetin and Sarul [11] for the blood bank location-allocation problem. A binary nonlinear goal programming model is formulated to minimise three objectives: the total fixed cost of location blood banks, the total distance travelled between blood banks and hospitals, and, unusually, an inequality index as a fairness mechanism for the distance. Sha and Huang [12] propose a multi-period location-allocation model with Lagrangian relaxation for solving an emergency blood supply problem.

Some research combines blood inventory issues with location problems. Motivated by distribution of perishable produce such as blood products, $\mathrm{Xu}$ [13] develops a joint inventory-location model for hospital blood banks. Total system costs are minimised: fixed costs, transportation costs and pooled inventory costs. A substitution algorithm is used to find solutions. A joint location-inventory problem with risk pooling is also considered by Shen et al. [14] who group hospitals and select one to be a regional blood centre for managing an inventory of platelets. The nonlinear integer programming model proposed is a modification of a set-covering model: it includes the inventory cost and the safety stock maintenance of the regional blood centres. Solutions are provided by a column generation algorithm.

Heuristics and meta-heuristics have been used to obtain results on large-sized healthcare facility location problems that cannot be solved using optimisation software. Valipour et al. [15] propose Improved Particle Swarm Optimization (IPSO) to maximise the population-to-healthcare-facility ratio within a coverage distance. Shariff et al. [16] present genetic algorithms to solve the Maximal Covering Location Problem for healthcare facility planning in Malaysia and compare results with those obtained using CPLEX.

\section{Model for location of low-cost blood facilities}

\subsection{Problem description}

The essence of the logistics problem of locating the low-cost collection and delivery centres is illustrated in Figure 3. Donors give blood either at one of the two types of blood service facilities proposed or at the NBC or RBCs. Transportation of whole blood and blood products is illustrated: all blood collected at a Type 1 donation centre goes straight to an allocated RBC. On the other hand, only samples of blood collected at Type 2 donation centres are sent to an RBC and results of screening and testing are reported online. After testing, safe blood products are distributed to demand points at hospitals. It should be noted that in this new supply chain, blood is collected only at the new collection facilities, as well as at RBCs and NBCs.

Locations for both types of blood service facilities are chosen according to a weighted sum of three criteria, of which the first is the estimated sum of donations at candidate sites. Secondly, total distances are summed from Type 1 and 2 facilities to an allocated RBC. Thirdly, total distances from RBCs or Type 2 distribution centres to hospital demand points are summed, weighted by estimated quantity of blood products transported. Criteria weights are supplied by the decision maker. The current NBC and RBC locations are excluded from the set of candidate sites for Type 1 and 2 facilities.

The model uses expected volumes of collection at each site for the planned time period, given historical data on local collections. The following assumptions are also made: that demand from hospitals can be 


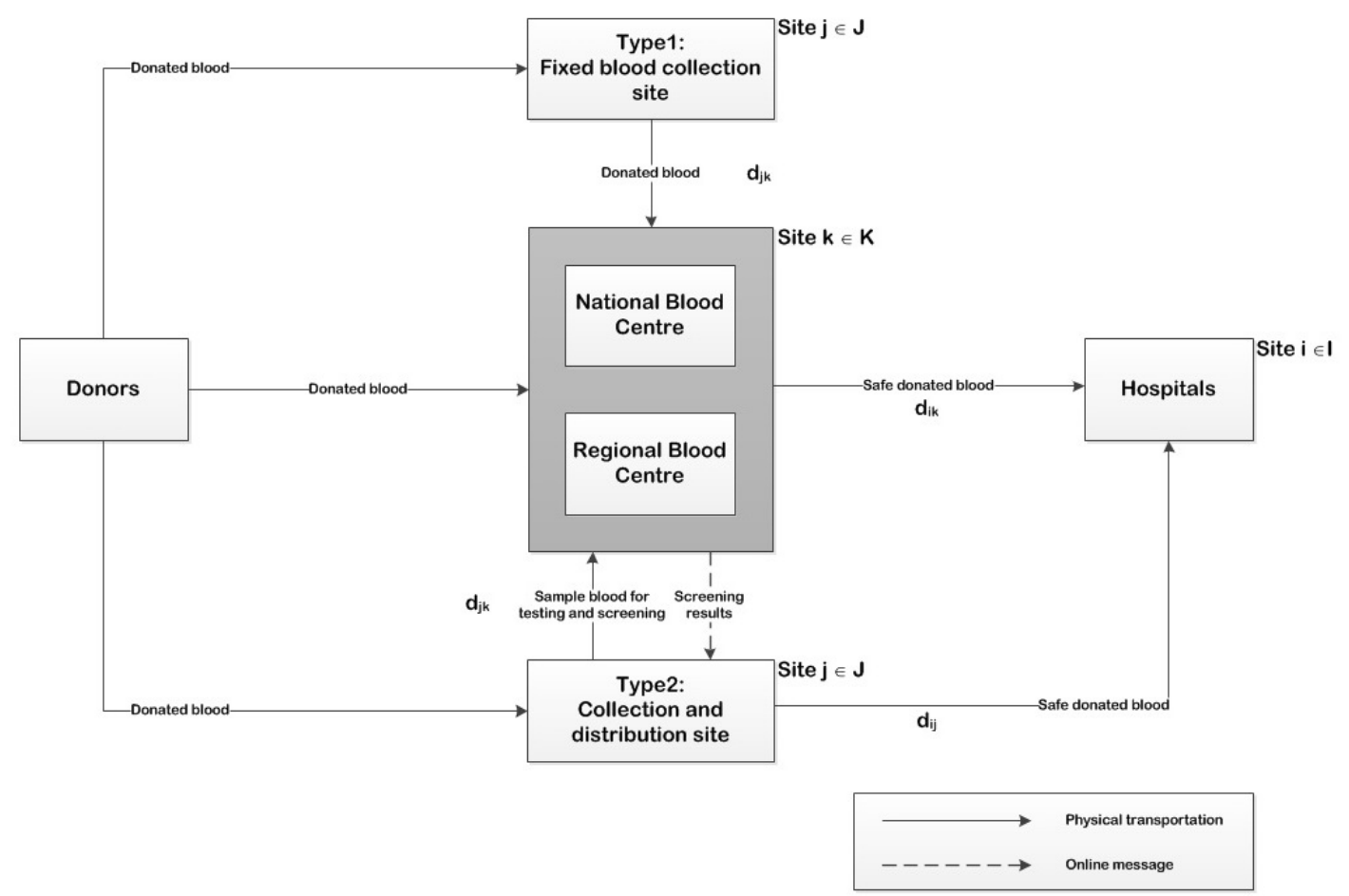

Figure 3: Proposed blood supply chain with two new types of blood service facilities

estimated, that a total budget for establishing the new facilities is known, and that the decision maker can weight the relative importance of improving supply against improving transportation costs. The costs of equipment may vary with the site of installation: this could be for reasons of the volumes of donations faced in a particular area. 


\subsection{Model formulation}

Notation
Symbol Description
I set of demand sites
J $\quad$ set of candidate sites for Type 1 and Type 2 facilities
$K \quad$ set of RBCs (including the NBC)
$b \quad$ budget allocated for establishing blood service facilities
$c_{k}^{1} \quad$ capacity of $\mathrm{RBC} k, k \in K$
$c_{j}^{2} \quad$ capacity of Type 2 facility at site $j, j \in J$
$d_{i k} \quad$ distance between the $i^{\text {th }}$ hospital and the $k^{\text {th }} \mathrm{RBC}, i \in I, k \in K$
$d_{j k} \quad$ distance between the $j^{\text {th }}$ facility site and the $k^{\text {th }} \mathrm{RBC}, j \in J, k \in K$
$d_{i j} \quad$ distance between the $j^{\text {th }}$ facility site and the $i^{\text {th }}$ hospital, $i \in \mathrm{I}, j \in J$
$e_{j}^{1}, e_{j}^{2} \quad$ equipment costs for Type 1 and 2 facilities at site $j, j \in J$
$f_{j}, f_{j}^{2} \quad$ construction costs for Type 1 and 2 facilities at site $j, j \in J$
$l_{j}^{1}, l_{j}^{2} \quad$ land costs for Type 1 and 2 facilities at site $j, j \in J$
$m$ maximum travel distance between a Type 2 facility and the regional blood centre
$s_{j} \quad$ annual expected amount of blood collected at the $j^{\text {th }}$ site in units of blood, $j \in J$
$u_{i} \quad$ average quantity of blood usage at the $i^{\text {th }}$ hospital in units of blood, $i \in I$
$w_{1}, w_{2}, w_{3} \quad$ weight score for the $1^{\text {st }}, 2^{\text {nd }}$ and $3^{\text {rd }}$ objective

Decision variables are as follows:

$$
\begin{aligned}
x_{i k}^{1} & = \begin{cases}1 & \text { if the } i^{t h} \text { hospital is allocated to the } k^{\text {th }} \mathrm{RBC}, i \in I, k \in K \\
0 & \text { otherwise }\end{cases} \\
x_{i j}^{2} & = \begin{cases}1 & \text { if the } i^{\text {th }} \text { hospital is allocated to a Type } 2 \text { facility at the } j^{\text {th }} \text { site, } i \in I, j \in J \\
0 & \text { otherwise }\end{cases} \\
y_{j}^{1} & = \begin{cases}1 & \text { if Type } 1 \text { facility is opened at the } j^{\text {th }} \text { site, } j \in J \\
0 & \text { otherwise }\end{cases} \\
y_{j}^{2} & = \begin{cases}1 & \text { if Type } 2 \text { facility is opened at the } j^{\text {th }} \text { site, } j \in J \\
0 & \text { otherwise }\end{cases} \\
z_{j k}^{1} & = \begin{cases}1 & \text { if Type } 1 \text { facility at the } j^{\text {th }} \text { site is allocated to the } k^{\text {th }} \mathrm{RBC}, j \in J, k \in K \\
0 & \text { otherwise }\end{cases} \\
z_{j k}^{2} & = \begin{cases}1 & \text { if Type } 2 \text { facility at the } j^{\text {th }} \text { site is allocated to the } k^{t h} \mathrm{RBC}, j \in J, k \in K \\
0 & \text { otherwise }\end{cases}
\end{aligned}
$$

\section{Model formulation}

The objective function (1) minimises the weighted sum of three components. The first component is the total travel distance from the two types of blood facilities to allocated RBCs. The second component is the 
demand-weighted distance from Type 2 facility or RBCs to allocated demand sites. The final component is the expected sum of blood donated during the planning period.

Minimise

$$
w_{1} \sum_{j \in J} \sum_{k \in K} d_{j k}\left(z_{j k}^{1}+z_{j k}^{2}\right)+w_{2} \sum_{i \in I} \sum_{k \in K} u_{i}\left(d_{i k} x_{i k}^{1}+d_{i j} x_{i j}^{2}\right)-w_{3}\left(\sum_{j \in J} s_{j}\left(y_{j}^{1}+y_{j}^{2}\right)\right)
$$

subject to

$$
\begin{gathered}
\sum_{k \in K} x_{i k}^{1}+\sum_{j \in J} x_{i j}^{2}=1, \quad \forall i \in I, \\
\sum_{k \in K} z_{j k}^{1}=y_{j}^{1}, \quad \forall j \in J, \\
\sum_{k \in K} z_{j k}^{2}=y_{j}^{2}, \quad \forall j \in J, \\
x_{i j}^{2} \leq y_{j}^{2}, \quad \forall i \in I, \quad \forall j \in J, \\
y_{j}^{1}+y_{j}^{2} \leq 1, \quad \forall j \in J, \\
z_{j k}^{1}\left(\frac{d_{j k}}{80}\right) \leq 4, \quad \forall j \in J, \quad \forall k \in K, \\
z_{j k}^{2} d_{j k} \leq m, \quad \forall j \in J, \quad \forall k \in K, \\
\sum_{j \in J}\left(e_{j}^{1}+f_{j}^{1}+l_{j}^{1}\right) y_{j}^{1}+\sum_{j \in J}\left(e_{j}^{2}+f_{j}^{2}+l_{j}^{2}\right) y_{j}^{2} \leq b, \\
\sum_{i \in I} u_{i} x_{i k}^{1} \leq c_{k}^{1}, \quad \forall k \in K, \\
\sum_{i \in I} u_{i} x_{i j}^{2} \leq c_{j}^{2}, \quad \forall j \in J, \\
z_{j k}^{1}, z_{i j}^{2} \in\{0,1\}, \quad \forall i \in I, \quad \forall j \in J, \quad \forall k \in K, \\
y_{j}^{1}, y_{j}^{2} \in\{0,1\}, \quad \forall j \in J, \\
z_{j k}^{2} \in\{0,1\}, \quad \forall j \in J, \quad \forall k \in K .
\end{gathered}
$$

Constraints (2) ensure that each hospital is allocated to exactly one RBC or one distribution centre. Constraints (3) and (4) allocate an open Type 1 or 2 facility $j$ to exactly one RBC $k$. Constraints (5) ensure that hospital $i$ is allocated only to an open Type 2 facility. Type 1 and Type 2 facilities cannot be opened at the same site via constraints (6). If Type 1 facility $i$ is allocated to RBC $k$, constraints (7) impose a maximum travel time of 4 hours on blood transported between the sites, assuming $80 \mathrm{kph}$ speed of travel. Constraints (8) define the maximum travel distance from Type 2 facility $j$ to the regional blood centre, $k$, to which it is allocated. Constraints (9) define the budgetary constraint, and constraints (10) and (11) give capacities at RBCs and Type 2 facilities. 


\section{Data used and results}

In this section we describe the data sources used for model runs and computational results obtained.

\section{Data sources}

A major data source used in this study was provided by the annual reports for years 1999 to 2009 of the National Blood Centre, Thai Red Cross Society [17]. Capitals of Thailand's 76 provinces were used as candidate sites for Type 1 or 2 facilities, unless RBCs or the NBC were already located there. From the available National Blood Centre data [17], it was observed that it is from the provincial capitals that most donations were received by the TRCS. Expected donations at each candidate site were calculated from the quantities given by existing donors who gave blood to local hospitals in that area if there was no RBC in the province [17].

The approximation was made that demand is concentrated in the capital of each the province. This aggregation enabled linear programming software to be used. All hospitals in one province are therefore assumed to be allocated to one distribution centre or blood centre.

Google Earth and GIS software ARCGIS version 10 were employed to compute distances among various nodes based on the national road network, as defined by a geographic data set of Thailand Transport Portal, Ministry of Transport in Thailand.

Costs for investment in facilities can be divided into three parts. Firstly, costs of land are different across the provinces, ranging from approximately 65,000 to $13,000,000$ baht per square feet. These are assessed by the Department of Land, Ministry of Interior. Secondly, the costs of equipment include beds for blood donation. In addition, Type 2 of blood service facilities must have refrigerators for storing blood at low temperatures to control its quality and blood cell separator machines for preparing blood and blood products. The costs for Type 2 facility equipment are seven times greater than costs for Type 1 . Lastly, the costs of construction vary depending on local labour costs in different areas, including the size of building. Type 2 facilities need more rooms available than Type 1 facilities.

Equally weighted objective components were used, after discussion with the National Blood Centre.

\section{Computational results and user interface}

In this section, we present the results based on the binary integer programming model implemented on real world data described. The proposed model has been solved using IBM ILOG CPLEX academic version 12.2 which is performed on $2.26 \mathrm{GHz}$ Intel Core 2 Duo CPU processor running on a Windows 7 operating system. The total time for computing is approximately 2.34 seconds.

The ILOG OPL program is embedded in Visual Basic.Net as the user interface of the application program shown in Figure 4. The decision maker can run different scenarios of budgetary constraints using the continuation feature of this screen.

\section{Recommended locations and insights obtained}

Runs were carried out assuming demand from 76 sites, i.e from the provincial capitals. These sites were also the candidate locations for Type 1 and Type 2 facilities.

One site in the northern region, Chiang Rai province, and one site in the southern region, Chumphon province, are recommended for Type 2 facilities, with distribution centres (see Figure 5), when budgets are higher than 90 million baht. Demand from several provinces is re-allocated to receive blood from the new distribution site. Hospitals in Chiang Rai, Nan and Phayao province are assigned to the distribution site at Chiang Rai province, while the distribution site at Chumphon province supports hospitals located at Chumphon, Ranong, and Prachuap Khiri Khan province.

Results of runs for budgets from 10 to 200 million baht are shown in Figure 6, which contrasts the decreasing costs of transporting blood to hospitals with increasing quantities of blood collected from donors. For lower budgets, up to 70 million baht, only the less costly Type 1 facilities are located, with rooms for donations only. At higher budgets, the more costly Type 2 facilities can also be afforded, with rooms for both donations and distribution. It can be noticed that there is a steady decline in the logistics 


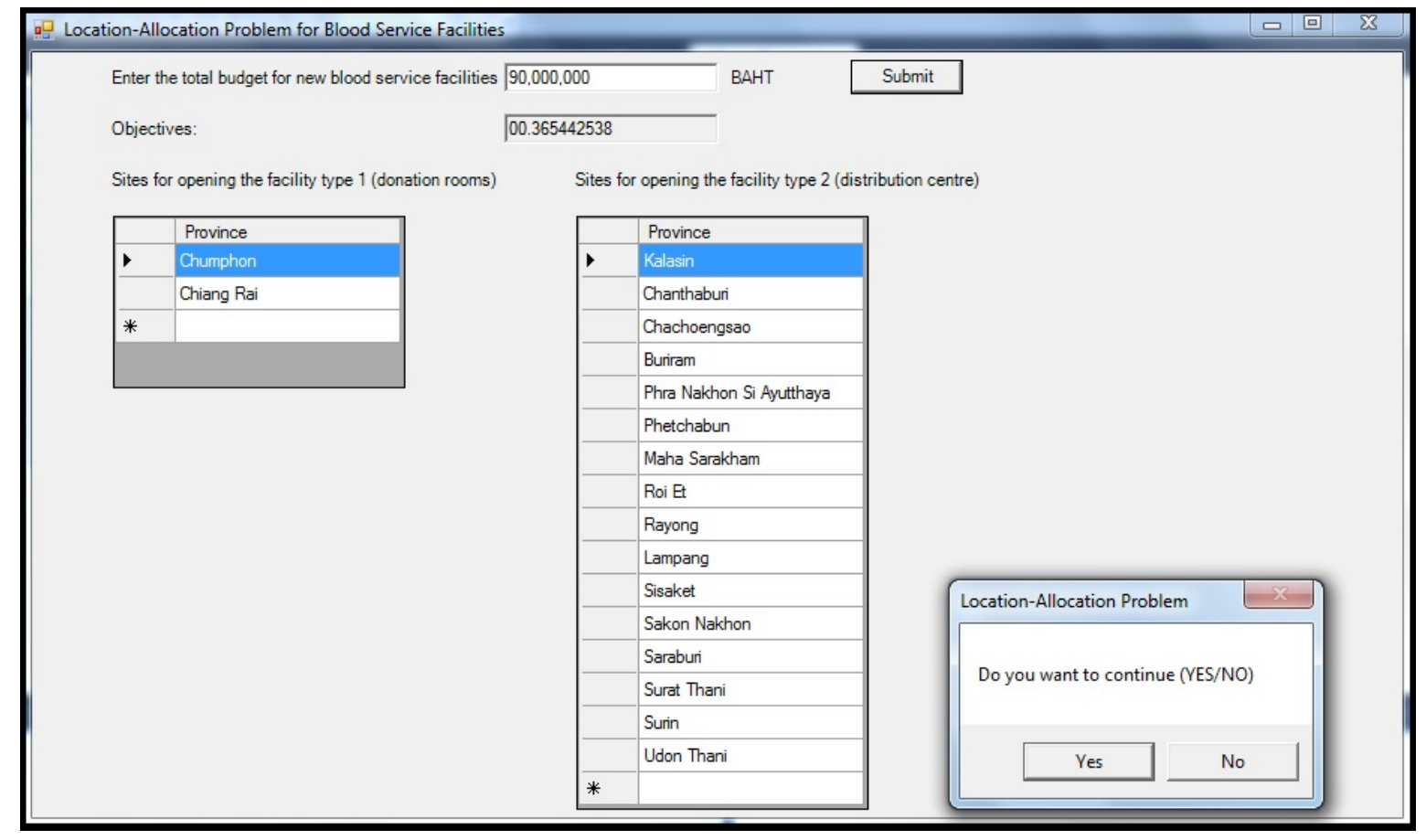

Figure 4: User interface for location-allocation model for low-cost blood service facilities

and transportation costs with the opening of a new distribution site. Figure 7 shows the numbers of Type 1 and Type 2 facilities sited according to a range of budget from 10 to 200 million baht.

Demand for blood products was assumed in model runs to be aggregated in the 76 provincial capitals (see Section 5.1). To determine the effects of this approximation, the actual locations of about 1000 hospitals were used in subsequent testing of results to compare current distances travelled to RBCs with distances to RBCs and the recommended Type 2 distribution facilities. Table 1 shows minimum, maximum, average, and standard deviations of actual travel distances obtained by allocating 1000 hospitals to the blood service centres. Results based on the proposed model show as improvement, with the overall average travelling distance for each visit from hospitals to RBCs or Type 2 distribution centres being reduced from $112 \mathrm{~km}$ to $101 \mathrm{~km}$. In most cases the average distance travelled from hospitals to individual RBCs is decreased by the new proposal. However, there are small increases to some RBCs and the NBC due to re-allocation of hospitals to even out travelling. 


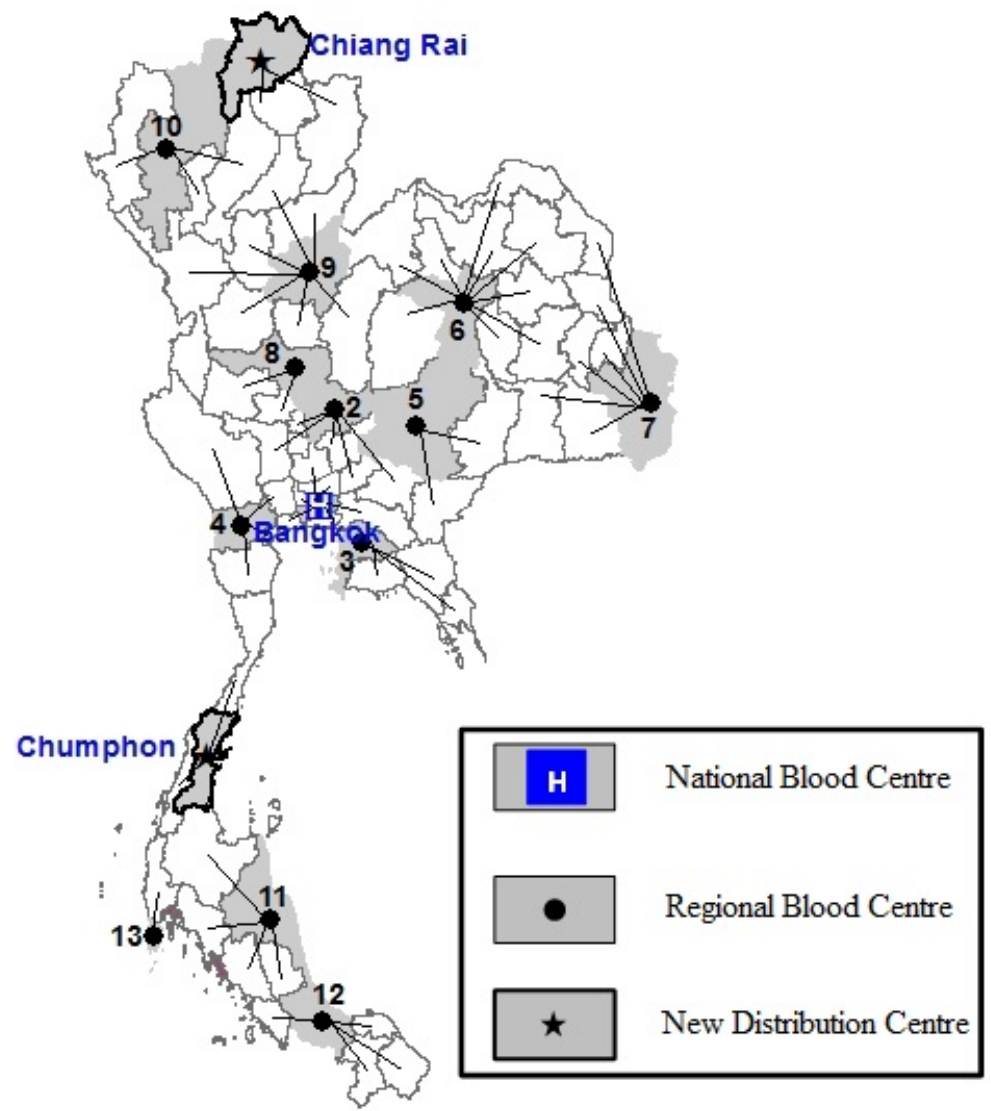

Figure 5: Locations recommended for Type 2 distribution sites with 90 million baht budget

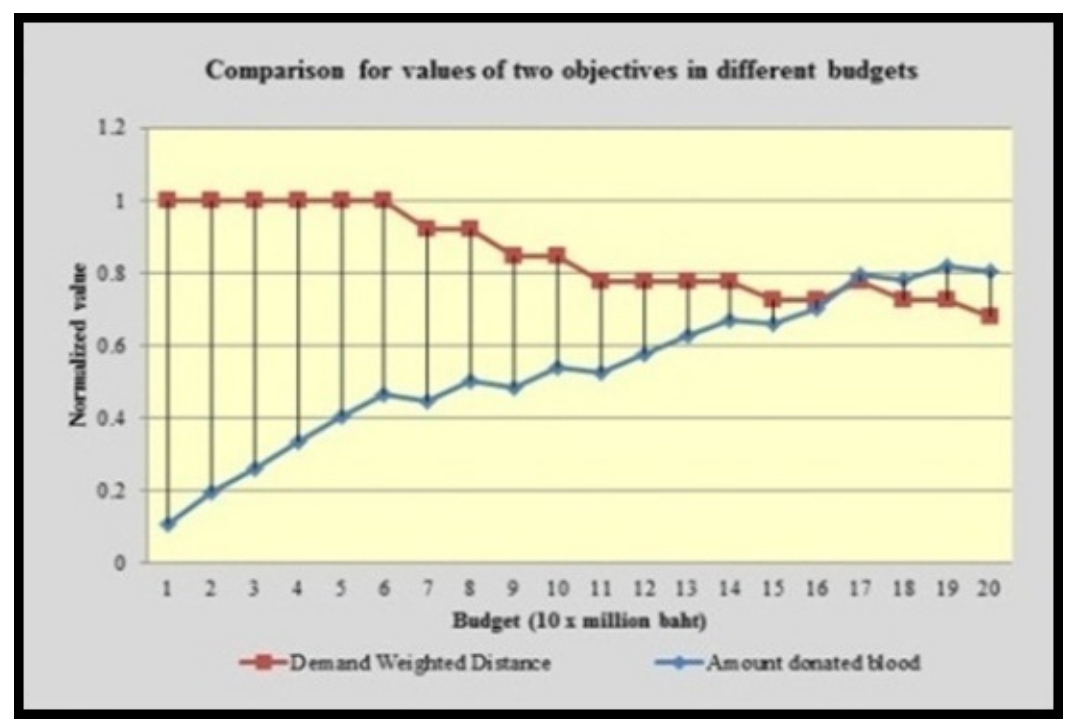

Figure 6: Comparing objectives: total demand-weighted distance vs blood donations collected 


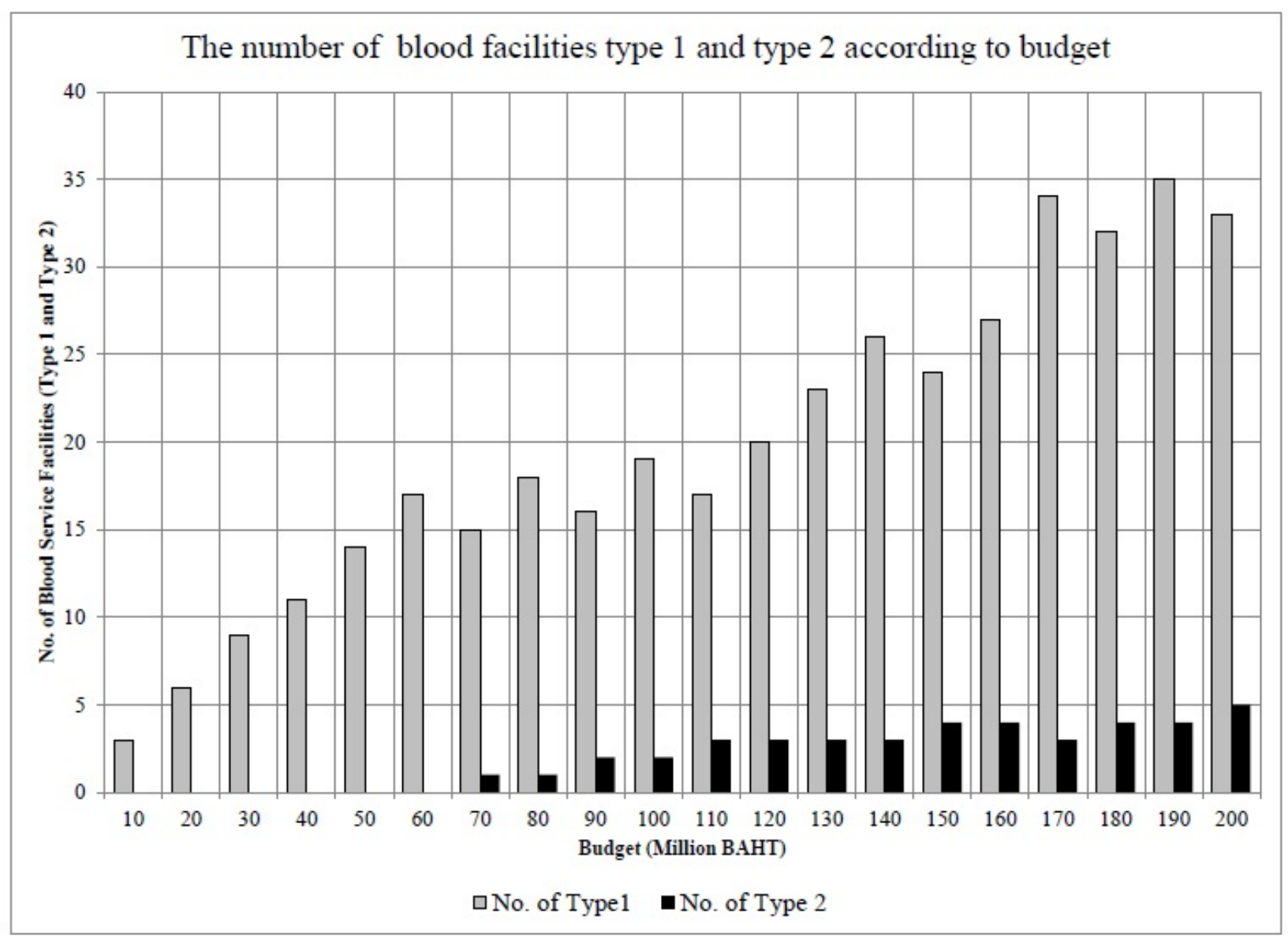

Figure 7: Comparing the number of Type 1 and Type 2 blood facilities by various budgets

\section{Discussion and conclusion}

Shortage of supply of safe blood products is a severe problem in many developing countries such as Thailand. In such regions, funds are limited for development of technologically advanced centres for blood screening and testing. However, low-cost solutions can be found that extend the reach of a network of existing blood centres. Increasing the number of blood services for collecting blood from donors in the provinces is expected to help increase amount of donated blood. This paper demonstrates the possibilities of improving the supply of blood products while also reducing transportation costs within available budgets. Binary Integer programming is proposed for this location-allocation problem based on contrasting objectives of improving supply while reducing transportation costs. Budgetary constraints take into account the full range of costs of construction of two types of facility, either donation room only or donation room with distribution centre.

Results of running different scenarios have been discussed with the TRCS. The TRCS's current plans for building blood distribution centres, based on working experience, propose siting a facility at Chiang Rai province, one of the sites recommended from model results. It is evident that two provinces, Nan and Phayao provinces, are currently far from the nearest RBC in Chiang Mai province. With re-allocation of demand from these provinces to Chiang Rai, transportation time for blood products to hospitals in these provinces can be reduced significantly. The results thus obtained from the model closely correspond with 


\begin{tabular}{|l|r|r|r|r|r|r|r|r|}
\hline \multirow{2}{*}{\multicolumn{1}{|c|}{ Blood Service Facility }} & \multicolumn{4}{|c|}{ Based on current situation } & \multicolumn{3}{c|}{ Based on solution proposed } \\
\cline { 2 - 8 } & \multicolumn{1}{|c|}{ Min. } & \multicolumn{1}{c|}{ Max. } & \multicolumn{1}{|c|}{ Avg. } & \multicolumn{1}{c|}{ Std. } & \multicolumn{1}{c|}{ Min. } & \multicolumn{1}{c|}{ Max. } & \multicolumn{1}{c|}{ Avg. } & \multicolumn{1}{c|}{ Std. } \\
\hline \hline NBC: Bangkok & 0.61 & 115.74 & 25.86 & 24.12 & 0.61 & 162.33 & 32.91 & 34.60 \\
RBC 2: Lopburi & 10.27 & 140.15 & 60.06 & 40.74 & 5.00 & 187.04 & 65.80 & 42.27 \\
RBC 3: Chonburi & 11.87 & 277.86 & 107.20 & 50.03 & 11.87 & 277.86 & 106.88 & 51.28 \\
RBC 4: Ratchaburi & 0.69 & 315.47 & 82.25 & 68.03 & 0.69 & 291.79 & 66.36 & 50.58 \\
RBC 5: Nakorn Ratchasima & 26.21 & 309.35 & 143.36 & 75.28 & 26.21 & 235.43 & 95.48 & 53.99 \\
RBC 6: Khon Kaen & 13.99 & 299.29 & 174.72 & 80.87 & 13.99 & 315.32 & 177.28 & 79.47 \\
RBC 7: Ubon Ratchathani & 23.00 & 315.96 & 179.53 & 81.80 & 23.00 & 315.96 & 179.53 & 81.80 \\
RBC 8: Nakorn Sawan & 28.89 & 381.13 & 140.23 & 88.95 & 28.89 & 155.47 & 74.46 & 32.64 \\
RBC 9: Phitsanulok & 2.24 & 370.34 & 139.45 & 98.71 & 2.24 & 315.76 & 117.90 & 63.26 \\
RBC 10: Chiang Mai & 3.26 & 289.28 & 116.45 & 56.71 & 32.26 & 289.28 & 110.81 & 60.04 \\
RBC 11: Nakorn Si Thammarat & 1.41 & 372.93 & 159.61 & 96.25 & 1.41 & 209.25 & 110.82 & 50.02 \\
RBC 12: Songkhla & 12.31 & 247.02 & 114.11 & 68.98 & 12.31 & 247.02 & 114.11 & 68.98 \\
RBC 13: Phuket & 5.38 & 18.74 & 14.48 & 4.68 & 5.38 & 114.50 & 41.58 & 36.56 \\
DC: Chiang Rai & & & & & 11.15 & 289.74 & 120.80 & 84.99 \\
DC: Chumphon & & & & & 11.82 & 263.66 & 97.15 & 74.61 \\
\hline \hline Overall average & - & - & 112.10 & - & - & - & 100.79 & - \\
\hline
\end{tabular}

Table 1: Comparison of distances for blood distribution to 1000 hospitals from the blood service facilities (unit: kilometre)

(NBC: National Blood Centre, RBC: Regional Blood Centre DC: Distribution Centre)

current opinion.

This study can be looked on as a short-term, low-cost solution to the problem of insufficient supplies in a resource-poor country. Operational costs, whether concerning labour costs at low-cost facilities or production costs at Regional Blood Centres, have not been made available to this study, which has considered only the major concerns of capital costs and transportation.

A direction for future research, should this be in line with TRCS plans, could be to consider phased-in development of facilities over a longer time period, say 20 years, when larger facilities might incur more economical running costs than the cheaper, smaller ones. Demand has been assumed to be aggregated in the provincial capitals. Future research could develop a heuristic method to enable increased numbers of demand points to be taken into account. The additional testing on travel distances to RBCs or proposed distribution centres shows that even with aggregated demand points, the modelling achieves improvements on the current situation. The solutions gained with more accurate locations for demand could be compared with those already obtained.

\section{References}

[1] R.L. Church, C.ReVelle, The maximal covering location problem, Papers of the Regional Science Association 32(1974) $101-118$.

[2] S.L. Hakimi, Optimum locations of switching centers and the absolute centers and medians of a graph, Oper. Res. 12(1964) 450-459.

[3] S.L. Hakimi, Optimum distribution of switching centers in a communication network and some related graph theoretic problems, Oper. Res. 13(1965) 462-475.

[4] H.K. Smith, P.R. Harper, C.N. Potts, Bicriteria efficiency/equity hierarchical location models for public service application, J. Oper. Res. Soc. 64(2013) 500-512.

[5] W.P. Pierskalla, Supply chain management of blood banks. In: M.L. Brandeau, F. Sainfort, W.P. Pierskalla, editors. Operations Research and Health Care, New York: Springer US;2005,p. 103-45.

[6] J. Rautonen, Redesigning supply chain management together with the hospitals. Transfusion 47(2007) 197-200.

[7] I. Or, W.P. Pierskalla, A transportation location-allocation model for regional blood banking, AIIE Transactions 11(1979) 86-95.

[8] D.A. Jacobs, M.N. Silan, B.A. Clemson, An analysis of alternative locations and srvice areas of American Red Cross blood facilities, Interfaces 26(1996) 40-50.

[9] M. Dessouky, F. Ordez, H. Jia, Z. Shen, Rapid distribution of medical supplies. In: R.W. Hall, editors. Patient Flow: Reducing Delay in Healthcare Delivery, New York: Springer US; 2006, p. 309-38. 
[10] G. Şahin, H. Sral, S. Meral, Locational analysis for regionalization of Turkish Red Crescent blood services, Comput. Oper. Res. 34(2007) 692-704.

[11] E. Çetin, L.S. Sarul, A blood bank location model: a multiobjective approach, Eur. J. Pure. Appl. Math. 2(2009) 112-124.

[12] Y. Sha, J. Huang, The multi-period location-allocation problem of engineering emergency blood supply systems, Syst. Eng. Proced. 5(2012) 21-28.

[13] P. Xu, Solving a joint inventory-location problem by a substitution algorithm. Industrial Engineering and Management Sciences Department Honors Project Report, Northwestern University 1999.

[14] Z.J.M. Shen, C. Coullard, M.S. Daskin, A joint location-inventory Model, Transp. Sci. 37(2003) 40-55.

[15] M.T. Valipour, A. Nedjati, R. Kazem, Solving health care facility location problems with new heuristic algorithm method, Intl. J. Modeling. Optim. 3(2013) 12-14.

[16] S.S.R. Shariff, N.H. Moin, M. Omar, Location allocation modeling for healthcare facility planning in Malaysia, Comput. Ind. Eng. 62(2012) 1000-1010.

[17] National Blood Centre, Thai Red Cross Society. Annual reports. Bangkok: Chulalongkorn University Printing House; $1999-2009$. 